\title{
Epidemiology, clinical profile and treatment patterns of venous thromboembolism in cancer patients in Taiwan: a population-based study
}

\author{
Tan-Wei Chew ${ }^{1}$, Churn-Shiouh Gau ${ }^{1,2,3}$, Yu-Wen Wen ${ }^{4}$, Li-Jiuan Shen ${ }^{1,2,5}$, C Daniel Mullins ${ }^{6}$ and Fei-Yuan Hsiao ${ }^{1,2,5^{*}}$
}

\begin{abstract}
Background: Venous thromboembolism (VTE) is a clinically significant complication that is well documented among Caucasian cancer patients. However, evidence regarding VTE incidence and treatment among Asian cancer patients is very limited. The objective of this study is to investigate the incidence, risk factors and management of VTE among Taiwanese cancer patients.

Methods: Using Taiwan's National Health Insurance Research Database, we identified 43,855 newly diagnosed cancer patients between 2001 and 2008. Two alternative algorithms for identifying VTE event were explored to better quantify a range of incidence rates of VTE in our cancer patients. Multivariable logistic regression models were used to explore VTE risk factors.

Results: The incidence rates of VTE were 9.9 (algorithm 1) and 3.4 (algorithm 2) per 1,000 person-years, respectively. The incidence rates were higher in certain cancers, particularly liver, pancreas, and lung. Significant risk factors for VTE were site of cancer, prior history of VTE, chemotherapy and major surgeries. Long-term anticoagulant therapy was initiated in $64.1 \%$ patients with VTE and $72.2 \%$ of them received warfarin alone. Approximately two-thirds of patients with VTE received $\leq 3$ months of anticoagulant therapy.
\end{abstract}

Conclusion: Incidence of cancer-related VTE is lower among Taiwanese compared to Caucasian populations. Nevertheless, risk factors for cancer-related VTE found in our study were consistent with current literature.

Keywords: Venous thromboembolism, Cancer, Epidemiology, Population-based study

\section{Background}

Venous thromboembolism (VTE) is a significant complication among cancer patients. The incidence rates of VTE among Caucasian cancer patients were reported to be 4-20\%. Cancer patients have 4- to 7-folds higher risk for VTE than the general population [1,2]. In addition, VTE-associated complications such as bleeding events, post-thrombotic syndrome and recurrence of VTE complicate the clinical management of cancer and worsen patients' quality of life [2]. Existing studies have further linked VTE to a higher risk of 1-year death post cancer diagnoses [3,4]. Several professional

\footnotetext{
* Correspondence: fyshsiao@ntu.edu.tw

'Graduate Institute of Clinical Pharmacy, College of Medicine, National Taiwan University, R220, 33, Linsen S. Road, Taipei 10050, Taiwan

${ }^{2}$ School of Pharmacy, College of Medicine, National Taiwan University, Taipei, Taiwan

Full list of author information is available at the end of the article
}

organizations, including American Society of Clinical Oncology (ASCO) and National Comprehensive Cancer Network (NCCN), have therefore issued guidelines regarding treatment and prophylaxis of VTE among cancer patients [5-9]. However, these guidelines were based on data mainly from Caucasian populations and their applications in different racial/ethnic populations are left unanswered.

In particular, available information on the epidemiology of VTE among Asian cancer patients is very limited. Although observational studies have tried to fill this knowledge gap, most existing studies were limited by small sample sizes and specific cancer sites [10-15]. Different methodological approaches may have contributed to dissimilar estimates of the incidence of VTE in the Asian cancer patients as well. Furthermore, treatment patterns for VTE among the Asian population may not 
follow clinical guidelines. A large scale, epidemiological study can help us to understand the incidence and treatment of VTE among Asian cancer patients and optimize clinical practice. Using a nationally-representative dataset, we conducted a population-based cohort study to investigate the epidemiology, risk factors, and clinical profile of VTE among Taiwanese cancer patients. In addition, we examined VTE treatment patterns in this population.

\section{Methods}

\section{Data source}

The data source of this population-based cohort study was the Taiwan's National Health Insurance research database (NHIRD). The NHIRD is a nationwide database comprising demographic data, clinical data, medical resource utilization data (outpatient and inpatient visits), costs of services, and treatment patterns of more than 99\% of the entire population ( 23 million) in Taiwan. All traceable personal identifiers are removed from the database to protect patient privacy. The database has been described in detail elsewhere [16]. The NHIRD has been maintained since 1997 and has been used to conduct many population-level studies $[16,17]$. Three subsets of the NHIRD, the Longitudinal Health Insurance Health Insurance Database 2000 (LHID 2000), 2005 (LHID 2005) and 2010 (LHID 2010), which contains claims data of one-million beneficiaries randomly selected from the Registry of Beneficiaries of the NHIRD in 2000, 2005, and 2010, respectively. The LHID 2000, LHID 2005, and LHID 2010 thus include approximately 15\% of the total population in Taiwan. The databases used in this study included all inpatient and outpatient medical claims of the LHID 2000, LHID 2005 and LHID 2010 from January, 1999 to December, 2009.

\section{Ethical statement}

Because the identification numbers for all of the subjects in the NHRID were encrypted to protect the privacy of the individuals, this study was exempt from a full review by the Institutional Review Board of the National Taiwan University Hospital and informed consent was waived.

\section{Study population}

Newly diagnosed cancer patients defined by those who have been first-ever hospitalized with a primary diagnosis of malignant disease (International Classification of Diseases, Ninth Revision, Clinical Modification (ICD-9CM) code (ICD-9-CM codes: 140-208)) between January 1, 2001 and December 31, 2008 were identified. A twoyear wash-out period was applied to ensure their incident diagnoses of cancer. The date when a patient was first hospitalized with a primary diagnosis of malignant disease was defined as the index date. Cancer subtypes analyzed in this study included head and neck (ICD-9CM codes: 140-149, 160-161), esophageal (150), stomach (151), colorectum (153-154), liver (155), pancreas (157), other abdominal $(152,156,158-159)$, lung (162163), sarcoma (170-171), skin (172-173), breast (174175), endometrium and cervix (179-182), ovary (183), prostate (185), testis (186), bladder (188), renal (189), brain (191-192), thyroid (193), non-Hodgkin's lymphoma (200, 202), Hodgkin's lymphoma (201), multiple myeloma (203), and leukemia (204-208). Patients were excluded if their genders were unknown. Those who had more than one primary diagnosis of malignant diseases at index date were also excluded, for their cancer sites cannot be categorized.

\section{Identification of VTE}

Two algorithms of VTE event were adopted in our study to better quantify a range of incidence rates of VTE in our cancer patients. VTE algorithm 1 was defined as a hospital admission with diagnostic codes of VTE (ICD9-CM codes 415.1x, 451.xx, 452, and 453.xx). VTE algorithm 2 was based on both the hospital admission with diagnostic codes of VTE and managements of VTE (prescription of intravenous or subcutaneous (IV/SC) anticoagulants (unfractionated heparin (UFH) or lowmolecular-weight heparin (LMWH)) or reimbursement codes of surgical thromboectomy) during the hospital stay.

\section{Comorbid diseases and potential risk factors for VTE}

Comorbid diseases, including hypertension, heart failure, ischemic heart disease, atrial fibrillation, renal insufficiency, liver disease, chronic lung disease, diabetes mellitus, stroke, rheumatologic diseases, varicose veins of lower extremities, degenerative and paralytic neurologic disease, peripheral vascular disease, anemia, arterial embolism and obesity, were retrieved from both the outpatient and inpatient medical claims for 1 year before or during the index date using relevant ICD-9 CM codes. A history of VTE was defined as being hospitalized with VTE diagnosis within 2 years before the index date.

Potential risk factors of VTE, including pregnancy, major surgery, hospitalization, cancer treatments (chemotherapy (including biologic therapy), radiation therapy, hormone therapy, and combination therapy), major extremity trauma, major spine trauma, blood transfusion, and infectious disease, were retrieved from inpatient or outpatient medical claims from 3 months before the VTE event to the end of follow-up date.

\section{Treatment pattern of VTE}

Among patients who had VTE events, we examined both initial and long-term treatment of VTE. Initial treatment patterns for patients who had VTE events were examined. 
Patients whose VTE events met VTE algorithm 2 were followed to see their long-term anticoagulant treatment pattern of VTE. Duration of long-term anticoagulant treatment was calculated from the discharge date of first hospitalization of VTE until the recurrence of VTE or end of follow-up date and was categorized into $\leq$ 3 months, 3-6 months, 6-12 months, and longer than 12 months.

\section{Statistical analysis}

Crude incidence rates of VTE for the entire cancer patients and subgroups of patients categorized by sites of cancer were calculated as the number of cases per 1,000 person-years. For VTE cases, the follow-up time started from index date to the date of VTE event. For patients without VTE event, the follow-up time started from index date to the end of follow-up. Comparisons between cancer patients with and without VTE were performed using Student's $t$-test for continuous variables and chi-square or Fisher's exact test for discrete variables.

Multivariable logistic regression models using stepwise selection were carried out to identify risk factors for VTE for the cohort defined using algorithm 2. To assess the association between cancer site and risk of VTE, we regrouped cancer sites as those with higher risk of VTE (GI tracts (stomach, colorectum, pancreas, liver, and esophagus), brain, lung, endometrium and cervix, ovary, and kidney) [1], hematological malignant diseases (nonHodgkin's lymphoma, Hodgkin's lymphoma, multiple myeloma, and leukemia) and other sites of cancer. Statistical significance was set at $\mathrm{p}<0.05$ and all tests were two-tailed. SAS software (Version 9.2; SAS Institute Inc., Cary, NC, USA) and Microsoft Office Excel 2010 were used in this study for the claims data conversion and analysis.

\section{Results \\ Baseline characteristics of study cohort and incidence rate of VTE}

We identified 43,855 newly diagnosed cancer patients between January 1, 2001 and December 31, 2008. The mean age $( \pm$ SD) of the study cohort was 59.5 years $( \pm 15.9$ years). Slightly more than half of them $(52.7 \%)$ were men and nearly forty percent $(41.9 \%)$ of patients were aged 65 years and older. Colorectal cancer (14.7\%) was the most frequently diagnosed cancer in our study cohort, followed by breast cancer (13.8\%), liver cancer (12.0\%), head and neck cancer (10.0\%), and lung cancer (9.5\%).

Among 43,855 newly diagnosed cancer patients, hospital admissions for VTE (algorithm 1) were identified in 1,388 patients $(3.2 \%)$ during or after index date. As shown in Table 1 , the overall incidence rate of VTE (algorithm 1) was 9.88 per 1,000 person-years. The incidence rates of VTE were higher in men than women (13.56 vs. 6.61 per 1,000 person-years). The incidence rates of VTE were higher in certain cancers, particularly cancer of liver (68.23 per 1,000 person-years), pancreas (27.83 per 1,000 person-years), lung (17.22 per 1,000 person-years), multiple myeloma (10.56 per 1,000 personyears), and non-Hodgkin's lymphoma (9.32 per 1,000 person-years). Taken together, these five cancers accounted for $67.6 \%$ of the VTE cases.

Hospital admissions for VTE (algorithm 2) were identified in 473 patients (1.1\%) during or after index date. The overall incidence rate of VTE (algorithm 2) was 3.35 per 1,000 person-years; slightly higher in men than women (3.89 vs. 2.86 per 1,000 person-years) (Table 1 ). The incidence rates of VTE were higher in certain cancers, particularly cancer of pancreas (16.05 per 1,000 person-years), lung (10.20 per 1,000 person-years), liver ( 9.06 per 1,000 person-years), multiple myeloma (7.92 per 1,000 person-years), and sarcoma (5.08 per 1,000 personyears). Taken together, these five cancers accounted for $42.3 \%$ of all VTE cases.

\section{Clinical characteristics of VTE events}

Most VTE events (VTE algorithm 1) (53.5\%) occurred within 90 days after index date, with $35.8 \%$ of VTE events occurring on the index date (Table 2). Median time-to-VTE was 70 days (range, $0-3,124$ days). Among patients who experience a VTE, the cumulative occurrence of VTE within 30, 90, 180, 270, and 365 days after index date were $42.9 \%, 53.5 \%, 61.8 \%, 66.3 \%$, and $70.8 \%$, respectively. Among patients hospitalized for VTE (algorithm 2), 59.4\% of VTE events occurred within 1 year after index date, with $18.0 \%$ of VTE events occurring on the index date (Table 2). Median time-to-VTE was 222 days (range, $0-3,124$ days). Cumulative occurrence of VTE within 30, 90, 180, 270, and 365 days after index date were $25.2 \%, 39.8 \%, 47.8 \%, 53.9$, and $59.4 \%$, respectively.

Anatomic distribution of VTE is shown in Table 3. Among 1,388 patients with VTE (algorithm 1), 9.7\% of patients had pulmonary embolism (PE) (with or without venous thrombosis) and $90.4 \%$ of patients had venous thrombosis. Among those with venous thrombosis, $52.9 \%$ had intra-abdominal thrombosis (thrombosis of renal, hepatic, or portal vein) and $27.7 \%$ had thrombosis of other unspecified site. Among 473 patients with VTE (algorithm 2), 16.1\% had PE (with or without venous thrombosis) and $80.7 \%$ had venous thrombosis. Among those with venous thrombosis, $19.0 \%$ had intra-abdominal thrombosis and $53.9 \%$ had thrombosis of unspecified site.

\section{Baseline characteristics and risk factors for VTE}

The mean age $( \pm$ SD) of cancer patients with VTE (algorithm 2) was 60.9 years ( \pm 14.3 years), which was 
Table 1 Site of cancer and associated incidence rate of VTE among all cancer patients

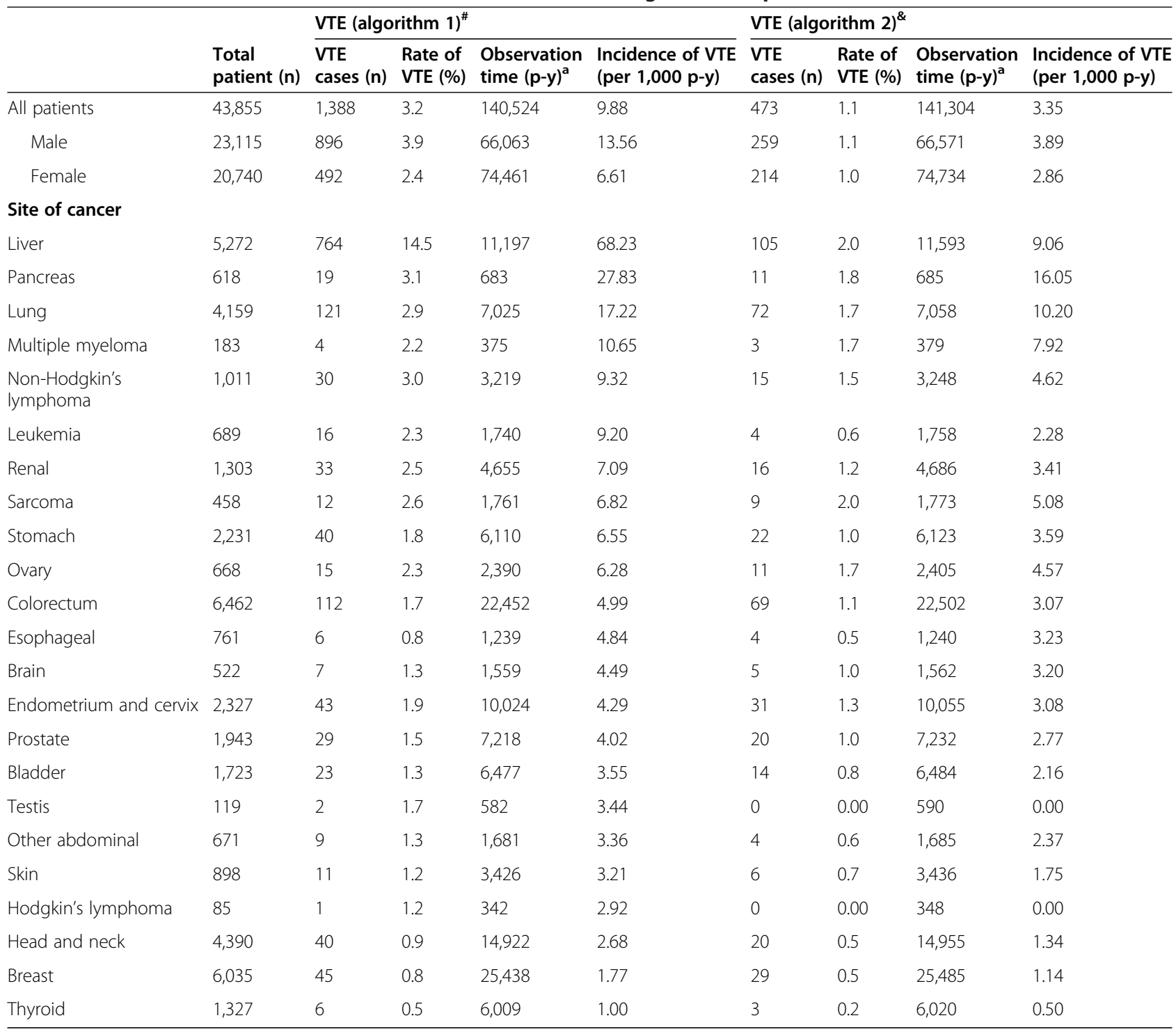

Abbreviations: $\mathrm{p}-\mathrm{y}$, person-years.

${ }^{a}$ For VTE cases, person-years were calculated from index date to the date of first hospitalization for VTE during or after cancer diagnosis. For patients without VTE event, person-years were calculated from index date until end of follow-up date.

"VTE algorithm 1 was defined as a hospital admission with diagnostic codes of VTE (ICD-9-CM codes: 415.1x, 451.xx, 452, and 453.xx).

\&VTE algorithm 2 was based on both the hospital admission with diagnostic codes of VTE and managements of VTE (prescription of intravenous or subcutaneous (IV/SC) anticoagulants (unfractionated heparin (UFH) or low-molecular-weight heparin (LMWH)) or reimbursement codes of surgical thromboectomy) during the hospital stay.

only slightly higher but statistically significantly different from cancer patients without VTE $(59.5 \pm 15.9$ years) (Table 4). Compared with cancer patients without VTE, more cancer patients with VTE had prior histories of VTE within the 2 years before index date $(1.1 \%$ vs. $0.2 \%$, $\mathrm{p}<0.0001$ ). Furthermore, patients with VTE were significantly more likely to have comorbid diseases (including hypertension, heart failure, ischemic heart disease, renal insufficiency, liver disease, rheumatologic diseases, arterial embolism, obesity, and varicose veins of lower extremities) than patients without VTE. Compared with patients without VTE, more patients with VTE received major surgery, active therapy, and G-CSF, or were diagnosed with infectious diseases within 3 months before/during the VTE event. Hospital admission was more frequent in patients with VTE $(60.0 \%$ vs. $32.1 \%, p<0.0001)$. Among patients who received active therapy, more patients with VTE received chemotherapy $(38.5 \%$ vs. $11.9 \%, \quad \mathrm{p}<0.0001)$ and combination therapy ( $4.2 \%$ vs. $1.2 \%, \mathrm{p}<0.0001)$, but more 
Table 2 Time-to-VTE after cancer diagnosis among all cancer patients

\begin{tabular}{|c|c|c|c|c|}
\hline \multirow[b]{2}{*}{ Time-to-VTE } & \multicolumn{2}{|c|}{ VTE algorithm $1^{\#}(\mathrm{~N}=1,388)$} & \multicolumn{2}{|c|}{ VTE algorithm $2^{\&}(\mathrm{~N}=473)$} \\
\hline & Patient no. (\%) & Cumulative rate of VTE (\%) & Patient no. (\%) & Cumulative rate of VTE (\%) \\
\hline 0 days & $497(35.8)$ & 35.8 & $85(18.0)$ & 18.0 \\
\hline $1-30$ days & $98(7.1)$ & 42.9 & $34(7.2)$ & 25.2 \\
\hline 31 - 90 days & $147(10.6)$ & 53.5 & $69(14.6)$ & 39.8 \\
\hline 91 - 180 days & $115(8.3)$ & 61.8 & $38(8.0)$ & 47.8 \\
\hline $181-270$ days & $63(4.5)$ & 66.3 & $29(6.1)$ & 53.9 \\
\hline $271-365$ days & $62(4.5)$ & 70.8 & $26(5.5)$ & 59.4 \\
\hline $366-545$ days & $70(5.0)$ & 75.8 & $32(6.8)$ & 66.2 \\
\hline $546-761$ days & $75(5.4)$ & 81.2 & $33(7.0)$ & 73.2 \\
\hline$>731$ days* & $261(18.8)$ & 100.0 & $127(26.9)$ & 100.0 \\
\hline
\end{tabular}

*The last observed events occurred 3,124 days after index date.

\#VTE algorithm 1 was defined as a hospital admission with diagnostic codes of VTE (ICD-9-CM codes 415.1x, 451.xx, 452, and 453.xx).

${ }^{\&}$ VTE algorithm 2 was based on both the hospital admission with diagnostic codes of VTE and managements of VTE (prescription of intravenous or subcutaneous (IV/SC) anticoagulants (unfractionated heparin (UFH) or low-molecular-weight heparin (LMWH)) or reimbursement codes of surgical thromboectomy) during the hospital stay.

patients without VTE received hormone therapy $(7.3 \%$ vs. $3.6 \%, \mathrm{p}<0.0001)$.

The results of multivariable logistic regression analysis showed that risk factors for VTE were primary cancer sites of GI, brain, lung, gynecologic, and renal, prior history of VTE, hypertension, arterial embolism, obesity, and rheumatologic diseases (Table 5). In addition, major thoracic, abdominal, and urogenital surgery, chemotherapy, and combination therapy were significantly associated with higher risk of VTE. In contrast, blood transfusion was associated with reduced risk of VTE.

\section{Treatment pattern of VTE}

Among 1,388 patients hospitalized for VTE (algorithm 1 ), only $33.6 \%$ of patients received anticoagulant therapy or surgical thromboectomy during the hospital stay. Only $7.9 \%$ of patients with thrombosis of hepatic, portal, or renal vein alone $(\mathrm{n}=734)$ received management of VTE. In contrast, excluding patients with superficial vein thrombosis $(n=4)$, anticoagulation or surgical thromboectomy was performed in $62.9 \%$ of patients with other sites of venous thrombosis or PE $(n=650)$. Among cancer patients with VTE events (algorithm 2), 1.5\% of

Table 3 Anatomic distribution of VTE among all cancer patients

\begin{tabular}{|c|c|c|}
\hline & $\begin{array}{l}\text { VTE algorithm } 1^{\#} \\
(\mathrm{~N}=1,388)\end{array}$ & $\begin{array}{l}\text { VTE algorithm } 2^{\&} \\
(\mathrm{~N}=473)\end{array}$ \\
\hline Sites & Patient no. (\%) & Patient no. (\%) \\
\hline Pulmonary embolism & $118(8.5)$ & $76(16.1)$ \\
\hline Pulmonary embolism and venous thrombosis & $16(1.1)$ & $15(3.2)$ \\
\hline Thrombosis of extremities & $73(5.3)$ & $19(4.0)$ \\
\hline Thrombosis of vena cava & $38(2.7)$ & $12(2.5)$ \\
\hline Thrombosis of renal vein, hepatic vein or portal vein & $734(52.9)^{a}$ & $90(19.0)^{b}$ \\
\hline Thrombosis of unspecified site & $384(27.7)$ & $255(53.9)$ \\
\hline Superficial venous thrombosis & $4(0.3)$ & $0(0.0)$ \\
\hline Multiple thrombotic sites & $21(1.5)^{c}$ & $6(1.3)^{d}$ \\
\hline
\end{tabular}

${ }^{a} 715$ patients had portal vein thrombosis, 12 patients had hepatic vein thrombosis, 7 patients had thrombosis of renal vein.

${ }^{b} 86$ patients had portal vein thrombosis, 2 patients had hepatic vein thrombosis, 2 patients had thrombosis of renal vein.

${ }^{c} 16$ patients had concomitant thrombosis of vena cava and intra-abdominal venous, 1 patient had concomitant thrombosis of extremities and other unspecified site, and 3 patients had concomitant thrombosis of vena cava, intra-abdominal venous and other unspecified site.

$\mathrm{d} 4$ patients had concomitant thrombosis of vena cava and intra-abdominal venous, and 2 patients had concomitant thrombosis of intra-abdominal venous and other unspecified site.

\#VTE algorithm 1 was defined as a hospital admission with diagnostic codes of VTE (ICD-9-CM codes 415.1x, 451.xx, 452, and 453.xx).

${ }^{\&}$ VTE algorithm 2 was based on both the hospital admission with diagnostic codes of VTE and managements of VTE (prescription of intravenous or subcutaneous (IV/SC) anticoagulants (unfractionated heparin (UFH) or low-molecular-weight heparin (LMWH)) or reimbursement codes of surgical thromboectomy) during the hospital stay. 
Table 4 Baseline characteristics of cancer patients with or without VTE

\begin{tabular}{|c|c|c|c|c|c|}
\hline & \multicolumn{2}{|c|}{ Patients without VTE } & \multicolumn{2}{|c|}{ VTE (algorithm $2^{\&}$ ) } & \multirow[t]{2}{*}{ P-value } \\
\hline & \multicolumn{2}{|c|}{$\mathrm{N}=43,382$} & \multicolumn{2}{|c|}{$\mathrm{N}=473$} & \\
\hline \multirow[t]{2}{*}{ Mean age (years) } & \multicolumn{2}{|c|}{$59.52 \pm 15.92$} & \multicolumn{2}{|c|}{$60.86 \pm 14.26$} & $0.0440^{*}$ \\
\hline & No. & $\%$ & No. & $\%$ & \\
\hline Age groups (years) & & & & & $0.0005^{*}$ \\
\hline$\leq 18$ & 502 & 1.2 & 1 & 0.2 & \\
\hline $19-40$ & 4,431 & 10.2 & 36 & 7.6 & \\
\hline $41-60$ & 16,847 & 38.8 & 179 & 37.9 & \\
\hline $61-80$ & 18,183 & 41.9 & 227 & 48.0 & \\
\hline$\geq 81$ & 3,419 & 7.9 & 30 & 6.3 & \\
\hline Gender & & & & & 0.3695 \\
\hline Male & 22,856 & 52.7 & 259 & 54.8 & \\
\hline Female & 20,526 & 47.3 & 214 & 45.2 & \\
\hline Prior history of VTE & 67 & 0.2 & 5 & 1.1 & $0.0011^{*}$ \\
\hline Hypertension & 15,981 & 36.8 & 223 & 47.2 & $<0.0001^{*}$ \\
\hline Heart failure & 1,793 & 4.1 & 31 & 6.6 & $0.0087^{*}$ \\
\hline Ischemic heart disease & 6,005 & 13.8 & 86 & 18.2 & $0.0066^{*}$ \\
\hline Atrial fibrillation & 649 & 1.5 & 9 & 1.9 & 0.4522 \\
\hline Renal insufficiency & 3,270 & 7.5 & 53 & 11.2 & $0.0027^{*}$ \\
\hline Chronic lung disease & 8,211 & 18.9 & 95 & 20.1 & 0.5229 \\
\hline Diabetes mellitus & 7,874 & 18.2 & 97 & 20.5 & 0.1861 \\
\hline Stroke & 2,690 & 6.2 & 30 & 6.3 & 0.8988 \\
\hline Degenerative \& paralytic neurologic disease & 3,665 & 8.5 & 47 & 9.9 & 0.2474 \\
\hline Rheumatologic diseases & 573 & 1.3 & 12 & 2.5 & $0.0218^{*}$ \\
\hline Liver disease & 7,959 & 18.4 & 119 & 25.2 & $0.0001^{*}$ \\
\hline Arterial embolism & 165 & 0.4 & 7 & 1.5 & $0.0028^{*}$ \\
\hline Anemia & 5,179 & 11.9 & 62 & 13.1 & 0.4354 \\
\hline Varicose veins of lower extremities & 170 & 0.4 & 5 & 1.1 & $0.0418^{*}$ \\
\hline Peripheral vascular disease & 515 & 1.2 & 9 & 1.9 & 0.1543 \\
\hline Pregnancy & 74 & 0.2 & 2 & 0.4 & 0.1979 \\
\hline Infectious diseases & 15,809 & 36.4 & 242 & 51.2 & $<0.0001^{*}$ \\
\hline Major trauma before VTE event & 1260 & 2.9 & 18 & 3.8 & 0.2465 \\
\hline Major spine trauma & 426 & 1.0 & 6 & 1.3 & 0.4775 \\
\hline Major extremity trauma & 861 & 2.0 & 12 & 2.5 & 0.3924 \\
\hline Major surgery before VTE event & 10,152 & 23.4 & 286 & 60.5 & $<0.0001^{*}$ \\
\hline CNS & 457 & 1.1 & 8 & 1.7 & 0.1779 \\
\hline Thorax & 4,706 & 10.9 & 158 & 33.4 & $<0.0001^{*}$ \\
\hline Abdomen & 6,094 & 14.1 & 167 & 35.3 & $<0.0001^{*}$ \\
\hline Urogenital & 1,397 & 3.2 & 43 & 9.1 & $<0.0001^{*}$ \\
\hline Orthopedic & 164 & 0.4 & 1 & 0.2 & 1.0000 \\
\hline Hospitalization & 13,909 & 32.1 & 284 & 60.0 & $<0.0001^{*}$ \\
\hline Blood transfusion & 3,665 & 8.5 & 49 & 10.4 & 0.1376 \\
\hline Active therapy & 9,079 & 20.9 & 221 & 46.7 & $<0.0001^{*}$ \\
\hline Chemotherapy only & 5,151 & 11.9 & 182 & 38.5 & $<0.0001^{*}$ \\
\hline Radiation only & 214 & 0.5 & 2 & 0.4 & 1.0000 \\
\hline
\end{tabular}


Table 4 Baseline characteristics of cancer patients with or without VTE (Continued)

\begin{tabular}{|c|c|c|c|c|c|}
\hline Hormone therapy only & 3,180 & 7.3 & 17 & 3.6 & $0.0020^{*}$ \\
\hline Combination therapy & 534 & 1.2 & 20 & 4.2 & $<0.0001^{*}$ \\
\hline Use of erythropoietin stimulating agent (ESA) & 828 & 1.9 & 9 & 1.9 & 0.9926 \\
\hline Use of granulocyte colony-stimulating factor (GCSF) & 1,282 & 3.0 & 41 & 8.7 & $<0.0001^{*}$ \\
\hline Thalidomide therapy & 27 & 0.1 & 0 & 0.0 & 1.0000 \\
\hline Obesity & 146 & 0.3 & 5 & 1.1 & $0.0243^{*}$ \\
\hline
\end{tabular}

*p-value $<0.05$.

${ }^{\&}$ VTE algorithm 2 was based on both the hospital admission with diagnostic codes of VTE and managements of VTE (prescription of intravenous or subcutaneous (IV/SC) anticoagulants (unfractionated heparin (UFH) or low-molecular-weight heparin (LMWH)) or reimbursement codes of surgical thromboectomy) during the hospital stay.

patients received thromboecytomy, and $98.5 \%$ of patients received LMWH/ UFH for initial treatment of VTE.

Patients with VTE (algorithm 2) were followed to analyze the long-term treatment of VTE. Among 473 patients with VTE (algorithm 2), 58 patients (12.3\%) did not have any medical claim in the database after the VTE event. We therefore explored patterns of long-term anticoagulant treatment in the remaining 415 patients. Overall, long-term anticoagulant therapy was initiated in $64.1 \%$ of patients (Table 6). Among them, $46.3 \%$ of patients received warfarin alone and $9.6 \%$ of patients received LMWH at any time. The median duration of anticoagulant therapy was 66 days (range, 2-1,442 days). Among these patients, $58.7 \%, 18.4 \%, 13.5 \%$ and $9.4 \%$ of them received $\leq 3$ months, 3-6 months, 6-12 months

Table 5 Multivariable logistic regression: risk factors for VTE among all cancer patients

\begin{tabular}{|c|c|c|}
\hline Variable & Odds ratio & $95 \% \mathrm{Cl}$ \\
\hline \multicolumn{3}{|l|}{ Cancer sites } \\
\hline \multicolumn{3}{|l|}{ Low risk (reference) } \\
\hline High risk & 1.63 & $1.31-2.02$ \\
\hline Hematologic & 1.26 & $0.79-2.00$ \\
\hline Prior history of VTE & 4.32 & $1.60-11.66$ \\
\hline \multicolumn{3}{|l|}{ Comorbid diseases } \\
\hline Hypertension & 1.41 & $1.17-1.70$ \\
\hline Arterial embolism & 2.96 & $1.31-6.67$ \\
\hline Obesity & 2.88 & $1.13-7.35$ \\
\hline Rheumatologic diseases & 1.90 & $1.05-3.43$ \\
\hline \multicolumn{3}{|l|}{ Potential risk factors } \\
\hline \multicolumn{3}{|l|}{ Surgery } \\
\hline Thoracic & 2.35 & $1.91-2.89$ \\
\hline Abdominal & 1.99 & $1.62-2.45$ \\
\hline Urogenital & 2.12 & $1.52-2.94$ \\
\hline Chemotherapy & 3.61 & $2.95-4.41$ \\
\hline Combination therapy & 4.95 & $3.08-7.96$ \\
\hline Blood transfusion & 0.57 & $0.42-0.77$ \\
\hline
\end{tabular}

and $\geq 12$ months of long-term anticoagulant therapy, respectively.

\section{Discussion}

Using the NHI research database, we examined the incidence, risk factors and clinical characteristics of VTE among patients with different cancer types over a period of 9 years. The major strength of our study is that we report population-based rates for VTE incidence and treatment patterns in an Asian population. Two alternative algorithm were used to capture VTE diagnosis codes and managements of VTE during the hospital stay. The second algorithm was similar to the outcome definition used in previous population-based studies [18-20]. To avoid serious underestimation of the incidence rate of VTE among cancer patients, we also included patients who hospitalized for VTE only (algorithm 1). By adopting two VTE algorithms in our study, we believe we could provide crude incidence rates of accidentally detected VTE and clinical symptomatic VTE.

In our study, $1.1 \%$ to $3.2 \%$ of all newly diagnosed cancer patients were hospitalized for VTE events, based on two algorithms of VTE. Our study showed a higher incidence of VTE among the non-Caucasian cancer patients, which is consistent with $\mathrm{Yu}$ et al. [20]. The incidence rate of VTE is 21- to 62- folds among cancer patients than the general population in Taiwan (15.9 per 100,000 person-years) [19]. Nevertheless, the VTE incidence is significantly lower than reports among Caucasian populations [21-23]. Among Caucasian patients with cancer, the estimated incidence rate of VTE ranges from $0.6 \%$ to $12.1 \%$ [24-31]. The incidence rate in our study is lower than reported rates of Cronin-Fenton et al. [29] and Khorana et al. [28] among Caucasian populations.

There is convincing evidence that the risk of VTE increases in proportion to the number of predisposing factors [32,33]. Identification of risk factors can help us to identify cancer patients with higher risk for VTE and optimize the prophylaxis of VTE. In our study, an increased risk of VTE was associated with cancer site, prior history of VTE, arterial embolism, hypertension, obesity, major surgery, chemotherapy, and combination 
Table 6 Initial and long-term treatment of VTE among all cancer patients

\begin{tabular}{|c|c|c|}
\hline & $\begin{array}{l}\left.\text { VTE (algorithm } 1^{\#}\right) \\
(\mathrm{N}=1,388)\end{array}$ & $\begin{array}{l}\left.\text { VTE (algorithm } 2^{\&}\right) \\
(\mathrm{N}=473)\end{array}$ \\
\hline Initial treatment & Patient no. (\%) & Patient no. (\%) \\
\hline Thromboectomy & $7(0.5)$ & $7(1.5)$ \\
\hline \multicolumn{3}{|c|}{ Low molecular weight heparin (LMWH) } \\
\hline LMWH alone & $58(4.2)$ & $64(13.5)$ \\
\hline LMWH + warfarin & $143(10.3)$ & $148(31.3)$ \\
\hline \multicolumn{3}{|l|}{ Unfractioned heparin (UFH) } \\
\hline UFH alone & $79(5.7)$ & $115(24.3)$ \\
\hline UFH + warfarin & $72(5.2)$ & $77(16.3)$ \\
\hline \multicolumn{3}{|l|}{$\mathrm{UFH}+\mathrm{LWMH}$} \\
\hline UFH + LMWH & $16(1.1)$ & $17(3.6)$ \\
\hline UFH + LMWH + warfarin & $43(3.1)$ & $45(9.5)$ \\
\hline Warfarin only & $49(3.5)$ & $0(0.00)$ \\
\hline No anticoagulation therapy & $921(66.4)$ & $0(0.00)$ \\
\hline Long-term treatment & Patient no. (\%) & Patient no. (\%) \\
\hline LMWH alone & - & $24(5.8 \%)$ \\
\hline UFH alone & - & $24(5.8 \%)$ \\
\hline Warfarin alone & - & $192(46.3 \%)$ \\
\hline LMWH + warfarin & - & $13(3.1 \%)$ \\
\hline UFH + warfarin & - & $10(2.4 \%)$ \\
\hline $\mathrm{LMWH}$ and UFH & - & $3(0.7 \%)$ \\
\hline No anticoagulant therapy & - & 149 (35.9\%) \\
\hline
\end{tabular}

\#VTE algorithm 1 was defined as a hospital admission with diagnostic codes of VTE (ICD-9-CM codes 415.1x, 451.xx, 452, and 453.xx).

${ }^{\&}$ VTE algorithm 2 was based on both the hospital admission with diagnostic codes of VTE and managements of VTE (prescription of intravenous or subcutaneous (IV/SC) anticoagulants (unfractionated heparin (UFH) or low-molecular-weight heparin (LMWH)) or reimbursement codes of surgical thromboectomy) during the hospital stay.

therapy. These results were consistent with existing studies among cancer patients $[25,27,28,31,34-36]$. Rheumatologic diseases were first identified as an independent risk factor for VTE among cancer patients in our study. Recent epidemiological studies among general population also suggested that certain rheumatologic diseases, including rheumatic arthritis (RA), dermatomyositis/ polymyositis and systemic lupus erythematous (SLE) were associated with increased risk of VTE $[37,38]$.

Thrombosis of portal vein is common in our study. In our study, 51.5\% (algorithm 1) and 18.2\% (algorithm 2) of patients with VTE had portal vein thrombosis alone. More than $80 \%$ of the portal vein thrombosis occurred in patients with liver cancer. Consistent with previous studies in Korea [12,13], we found that most patients with thrombosis of renal, portal or hepatic veins did not received anticoagulant treatment in clinical practice. Liver cancer is a well-known risk factor for portal vein thrombosis $[39,40]$. The high prevalence of liver cancer in Taiwan [41] may result in the high prevalence of portal vein thrombosis in our study cohort. However, there is no randomized trial to guide the use of anticoagulation in this situation $[39,42]$. Given the common prevalence of portal vein thrombosis, further studies are needed to clarify the role and duration of anticoagulant therapy in these patients.

This study represents the largest national populationbased epidemiologic study in Asia that described the management of VTE among cancer patients in Taiwan. Our study found that the adherence to treatment guidelines was poor in Taiwan. Long-term anticoagulant therapy was only initiated in $64.1 \%$ of patients with VTE (algorithm 2). In existing clinical guidelines, 3-6 months of LMWH are recommended for long-term treatment of VTE in cancer patients $[1,6,7,42]$. However, in our study, among patients who received long-term treatment of VTE, LMWH was administered to only 9.6\% of patients at anytime following the VTE events. Most patients received warfarin monotherapy for the long-term treatment of VTE. This is probably because reimbursement for outpatient use of LMWH by NHI was limited to pregnant patients with prosthetic valve 
replacement [20]. Furthermore, in our study, treatment duration of long-term anticoagulant therapy was shorter than those recommended in clinical guidelines $[1,6,7,42]$.

Some limitations exist in the present study, generally related to the use of a claims database. First, the actual incidence of VTE may be underestimated, because some patients with PE die suddenly without accurate diagnosis. Patients who treated in the outpatient clinic using LMWH/oral anticoagulants were not included based on our definitions. Identification of a VTE event based on admission record may underestimate the incidence of VTE. However, the magnitude of underestimation could be very small as home injection of LMWH/UFH/fondapariunx is not reimbursed under Taiwan's National Health Insurance system, cancer patients usually are admitted for receiving these treatments. In addition, as patients usually receive LMWH/UFH for the initial treatment of VTE ("incident" VTE in our study) and warfarin for the long-term treatment, it is easier for physicians to monitor activated partial thromboplastin time (aPTT) and international normalized ratio (INR) when patients are hospitalized. These could be supported by another epidemiological study of VTE in the Taiwanese general population, in which the authors define their VTE cases as those who admitted for a VTE event [19]. Furthermore, patients with asymptomatic VTE may not be documented. We were unable to distinguish symptomatic VTE and incidentally detected VTE as the information were not routinely captured in a claim database. However, our estimates provide the incidence rate of clinically overt VTE, which is useful in helping decision making of VTE prophylaxis in clinical practice. A second limitation of our study is we identified our cancer cases based on a diagnosis of cancer at hospitalization, cancer cases diagnosed based on outpatient visit were not included, which may underestimate the cancer population. Nevertheless, as all NHI beneficiaries diagnosed with cancer is required to have an confirmed cancer diagnosis from the hospital to be eligible for a Certificate of Catastrophic Illness to be exempted from all co-payments, cancer cases who only be treated in outpatient setting are very few in Taiwan.

This approach could thus help us precisely identify the cancer cases and avoid potential misclassifications. Third, other factors that may contribute to development of VTE, including disease stage, laboratory data and performance status could not be obtained from the databases. However, we include many variables such as active therapy (e.g. radiation therapy) to serve as the proxy of disease severity to reduce potential confounding effects. Fourth, the study cohort may have received anticoagulant treatment based on certain baseline and prognostic characteristics. Finally, thromboprophylaxis can alter the incidence of VTE, but its use in our study population is unknown.

\section{Conclusion}

In conclusion, this retrospective cohort study describes the epidemiology, risk factors, and clinical profile of VTE across different types of cancer among an Asian population. The incidence rate of VTE was higher among cancer patients than among non-cancer patients in Taiwan but lower than among Caucasian cancer patients. Clinical practitioners should carefully monitor patients with cancer for VTE. Adherence to treatment guidelines was low in this real world cohort of Taiwanese cancer patients with VTE. Treatment and prophylaxis of VTE should be optimized, especially in patients with higher-risk of VTE. Due to the different epidemiologic profile of VTE in Taiwan compared with Caucasian population, further investigations are desired to estimate the harms and benefits of anticoagulants treatment and thromboprophylaxis among Asian cancer patients.

\section{Competing interests}

The authors declare that they have no competing interests.

\section{Authors' contributions}

HFY and MCD designed the research and wrote the paper. CTW analyzed data and wrote the paper. GCS and SLJ help perform the research. WYW contributed vital analytical tools. All authors read and approved the final manuscript

\section{Acknowledgements}

We thank the National Health Insurance Administration (NHIA) and National health Research Institutes (NHRI) for making available the databases for this study. Dr. Hsiao FY received research assistantships from a research project (NSC102-2410-H-002-058-MY2) sponsored by National Science Council, Taiwan and a research project (MOHW103-FDA-41100) sponsored by Food and Drug Administration (FDA), Taiwan. The content of this article, however, in no way represents any official position of the NHIA or NHRI. The author had full access to all the data in the study and takes responsibility for the integrity of the data and the accuracy of the data analysis. We thank Dr. Shao C. Chiang of the Koo Foundation Sun Yat-Sen Cancer Center, Taiwan, for providing consultation and assistance with the revised manuscript.

\section{Author details}

${ }^{1}$ Graduate Institute of Clinical Pharmacy, College of Medicine, National Taiwan University, R220, 33, Linsen S. Road, Taipei 10050, Taiwan. ${ }^{2}$ School of Pharmacy, College of Medicine, National Taiwan University, Taipei, Taiwan. ${ }^{3}$ Center for Drug Evaluation, Taipei, Taiwan. ${ }^{4}$ Clinical Informatics and Medical Statistics Research Center, Chang Gung University, Tao-Yuan, Taiwan. ${ }^{5}$ Department of Pharmacy, National Taiwan University Hospital, Taipei, Taiwan. ${ }^{6}$ University of Maryland School of Pharmacy, Baltimore, MD, USA.

Received: 4 September 2013 Accepted: 17 March 2015

Published online: 17 April 2015

\section{References}

1. Lyman GH, Khorana AA, Falanga A, Clarke-Pearson D, Flowers C, Jahanzeb $M$, et al. American Society of Clinical Oncology guideline: recommendations for venous thromboembolism prophylaxis and treatment in patients with cancer. J Clin Oncol. 2007;25:5490-505.

2. Lyman $\mathrm{GH}$. Venous thromboembolism in the patient with cancer: focus on burden of disease and benefits of thromboprophylaxis. Cancer. 2011;117:1334-49. 
3. Chew HK, Wun T, Harvey DJ, Zhou H, White RH. Incidence of venous thromboembolism and the impact on survival in breast cancer patients. J Clin Oncol. 2007;25:70-6.

4. Chew HK, Davies AM, Wun T, Harvey D, Zhou H, White RH. The incidence of venous thromboembolism among patients with primary lung cancer. J Thromb Haemost. 2008;6:601-8.

5. Lyman GH, Kuderer NM. Prevention and treatment of venous thromboembolism among patients with cancer: the American Society of Clinical Oncology Guidelines. Thromb Res. 2010;125 Suppl 2:S120-7.

6. Mandala M, Falanga A, Roila F. Management of venous thromboembolism (VTE) in cancer patients: ESMO Clinical Practice Guidelines. Ann Oncol. 2011;22(6):vi85-92.

7. National Comprehensive Cancer Network Web site. NCCN clinical pratice guidelines in oncology: Venous Thromboembolic Disease v.2.2011. (Accessed March 30, 2012, at www.nccn.org).

8. Farge D, Bosquet L, Kassab-Chahmi D, Mismetti P, Elalamy I, Meyer G, et al. 2008 French national guidelines for the treatment of venous thromboembolism in patients with cancer: report from the working group. Crit Rev Oncol Hematol. 2010;73:31-46.

9. Debourdeau P, Kassab Chahmi D, Le Gal G, Kriegel I, Desruennes E, Douard MC, et al. 2008 SOR guidelines for the prevention and treatment of thrombosis associated with central venous catheters in patients with cancer: report from the working group. Ann Oncol. 2009;20:1459-71.

10. Oh SY, Kim JH, Lee KW, Bang SM, Hwang JH, Oh D, et al. Venous thromboembolism in patients with pancreatic adenocarcinoma: lower incidence in Asian ethnicity. Thromb Res. 2008;122:485-90.

11. Koh Y, Bang SM, Lee JH, Yoon HJ, Do YR, Ryoo HM, et al. Low incidence of clinically apparent thromboembolism in Korean patients with multiple myeloma treated with thalidomide. Ann Hematol. 2010;89:201-6.

12. Lee KW, Bang SM, Kim S, Lee HJ, Shin DY, Koh Y, et al. The incidence, risk factors and prognostic implications of venous thromboembolism in patients with gastric cancer. J Thromb Haemost. 2010;8:540-7.

13. Choi S, Lee KW, Bang SM, Kim S, Lee JO, Kim YJ, et al. Different characteristics and prognostic impact of deep-vein thrombosis/pulmonary embolism and intraabdominal venous thrombosis in colorectal cancer patients. Thromb Haemost. 2011;106:1084-94.

14. Yokoyama K, Murata M, Ikeda Y, Okamoto S. Incidence and risk factors for developing venous thromboembolism in Japanese with diffuse large b-cell lymphoma. Thromb Res. 2012;130:7-11.

15. Kang MJ, Ryoo BY, Ryu MH, Koo DH, Chang HM, Lee JL, et al. Venous thromboembolism (VTE) in patients with advanced gastric cancer: an Asian experience. Eur J Cancer. 2012;48:492-500.

16. Hsiao FY, Yang CL, Huang YT, Huang WF. Using Taiwan National Health Insurance Research Databases for pharmacoepidemiology research. J Food Drug Anal. 2007;15:99-108.

17. Chen YC, Yeh HY, Wu JC, Haschler I, Chen TJ, Wetter T. Taiwan's National Health Insurance Research Database: administrative health care database as study object in bibliometrics. Scientometrics. 2011;86:365-80.

18. Jang MJ, Bang SM, Oh D. Incidence of venous thromboembolism in Korea: from the Health Insurance Review and Assessment Service database. J Thromb Haemost. 2011;9:85-91.

19. Lee $\mathrm{CH}$, Lin $L$ J, Cheng $\mathrm{CL}$, Kao Yang YH, Chen JY, Tsai LM. Incidence and cumulative recurrence rates of venous thromboembolism in the Taiwanese population. J Thromb Haemost. 2010;8:1515-23.

20. Yu YB, Gau JP, Liu CY, Yang MH, Chiang SC, Hsu HC, et al. A nation-wide analysis of venous thromboembolism in 497,180 cancer patients with the development and validation of a risk-stratification scoring system. Thromb Haemost. 2012;108(2):225-35.

21. White $\mathrm{RH}$, Zhou H, Murin S, Harvey D. Effect of ethnicity and gender on the incidence of venous thromboembolism in a diverse population in California in 1996. Thromb Haemost. 2005;93:298-305.

22. White $\mathrm{RH}$, Keenan $\mathrm{CR}$. Effects of race and ethnicity on the incidence of venous thromboembolism. Thromb Res. 2009;123 Suppl 4:S11-7.

23. Stein PD, Kayali F, Olson RE, Milford CE. Pulmonary thromboembolism in Asians/Pacific Islanders in the United States: analysis of data from the National Hospital Discharge Survey and the United States Bureau of the Census. Am J Med. 2004;116:435-42.

24. Levitan N, Dowlati A, Remick SC, Tahsildar HI, Sivinski LD, Beyth R, et al. Rates of initial and recurrent thromboembolic disease among patients with malignancy versus those without malignancy. Risk analysis using Medicare claims data. Medicine. 1999;78:285-91.
25. Sallah S, Wan JY, Nguyen NP. Venous thrombosis in patients with solid tumors: determination of frequency and characteristics. Thromb Haemost. 2002:87:575-9.

26. Stein PD, Beemath A, Meyers FA, Skaf E, Sanchez J, Olson RE. Incidence of venous thromboembolism in patients hospitalized with cancer. Am J Med. 2006:119:60-8.

27. Khorana AA, Francis CW, Culakova E, Fisher RI, Kuderer NM, Lyman GH. Thromboembolism in hospitalized neutropenic cancer patients. J Clin Oncol. 2006;24:484-90.

28. Khorana AA, Francis CW, Culakova E, Kuderer NM, Lyman GH. Frequency, risk factors, and trends for venous thromboembolism among hospitalized cancer patients. Cancer. 2007;110:2339-46.

29. Cronin-Fenton DP, Sondergaard F, Pedersen LA, Fryzek JP, Cetin K, Acquavella J, et al. Hospitalisation for venous thromboembolism in cancer patients and the general population: a population-based cohort study in Denmark, 1997-2006. Br J Cancer. 2010;103:947-53.

30. Khorana AA, Francis CW, Culakova E, Lyman GH. Risk factors for chemotherapy-associated venous thromboembolism in a prospective observational study. Cancer. 2005;104:2822-9.

31. Shah MA, Capanu M, Soff G, Asmis T, Kelsen DP. Risk factors for developing a new venous thromboembolism in ambulatory patients with nonhematologic malignancies and impact on survival for gastroesophageal malignancies. J Thromb Haemost. 2010;8:1702-9.

32. Anderson Jr FA, Wheeler HB. Physician practices in the management of venous thromboembolism: a community-wide survey. J Vasc Surg. 1992;16:707-14.

33. Anderson Jr FA, Spencer FA. Risk factors for venous thromboembolism. Circulation. 2003;107:19-16.

34. Blom JW, Vanderschoot JP, Oostindier MJ, Osanto S, Van der Meer FJ, Rosendaal FR. Incidence of venous thrombosis in a large cohort of 66,329 cancer patients: results of a record linkage study. J Thromb Haemost. 2006;4:529-35.

35. Agnelli G, Bolis G, Capussotti L, Scarpa RM, Tonelli F, Bonizzoni E, et al. A clinical outcome-based prospective study on venous thromboembolism after cancer surgery: the @RISTOS project. Ann Surg. 2006;243:89-95.

36. Hall IE, Andersen MS, Krumholz HM, Gross CP. Predictors of venous thromboembolism in patients with advanced common solid cancers. J Cancer Epidemiol. 2009;2009:182521.

37. Matta F, Singala R, Yaekoub AY, Najjar R, Stein PD. Risk of venous thromboembolism with rheumatoid arthritis. Thromb Haemost. 2009;101:134-8.

38. Ramagopalan SV, Wotton CJ, Handel AE, Yeates D, Goldacre MJ. Risk of venous thromboembolism in people admitted to hospital with selected immune-mediated diseases: record-linkage study. BMC Med. 2011;9:1.

39. Parikh S, Shah R, Kapoor P. Portal vein thrombosis. Am J Med. 2010;123:111-9.

40. Ponziani FR, Zocco MA, Campanale C, Rinninella E, Tortora A, Di Maurizio L, et al. Portal vein thrombosis: insight into physiopathology, diagnosis, and treatment. World J Gastroenterol. 2010;16:143-55.

41. Cancer Registry Annual Report, 2008, Taiwan. (Accessed 13 June, 2012, at http://tcr.cph.ntu.edu.tw/main.php?Page=N2).

42. Kearon C, Akl EA, Comerota AJ, Prandoni P, Bounameaux H, Goldhaber SZ, et al. Antithrombotic therapy for VTE disease: Antithrombotic Therapy and Prevention of Thrombosis, 9th ed: American College of Chest Physicians Evidence-Based Clinical Practice Guidelines. Chest. 2012;141:e419S-94S.

\section{Submit your next manuscript to BioMed Central and take full advantage of:}

- Convenient online submission

- Thorough peer review

- No space constraints or color figure charges

- Immediate publication on acceptance

- Inclusion in PubMed, CAS, Scopus and Google Scholar

- Research which is freely available for redistribution 